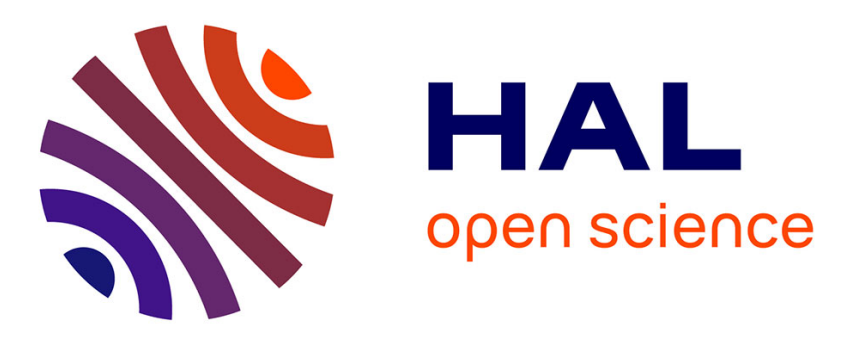

\title{
Essais d'acclimatation des arbres à quinquina en Indochine. Sixième note
}

Alexandre Yersin

\section{To cite this version:}

Alexandre Yersin. Essais d'acclimatation des arbres à quinquina en Indochine. Sixième note. Revue de botanique appliquée et d'agriculture coloniale, 1939, 212, pp.237-242. pasteur-00538680

\section{HAL Id: pasteur-00538680}

https://hal-pasteur.archives-ouvertes.fr/pasteur-00538680

Submitted on 23 Nov 2010

HAL is a multi-disciplinary open access archive for the deposit and dissemination of scientific research documents, whether they are published or not. The documents may come from teaching and research institutions in France or abroad, or from public or private research centers.
L'archive ouverte pluridisciplinaire HAL, est destinée au dépôt et à la diffusion de documents scientifiques de niveau recherche, publiés ou non, émanant des établissements d'enseignement et de recherche français ou étrangers, des laboratoires publics ou privés. 


\section{REVUE \\ de \\ Botanique Appliquée et \\ D'AGRICULTURE TROPICALE}

Publiée sous la Direction du

\section{Prof. Aug. CHEVALIER}

Professeur au Muséum natıonal d'Histoire naturelle de Paris,

Chef de la Mission permanente d'Etudes des Cultures et Jardins d'essais coloniaux,

Secrétaire Général de l'Association Colonies-Sciences.

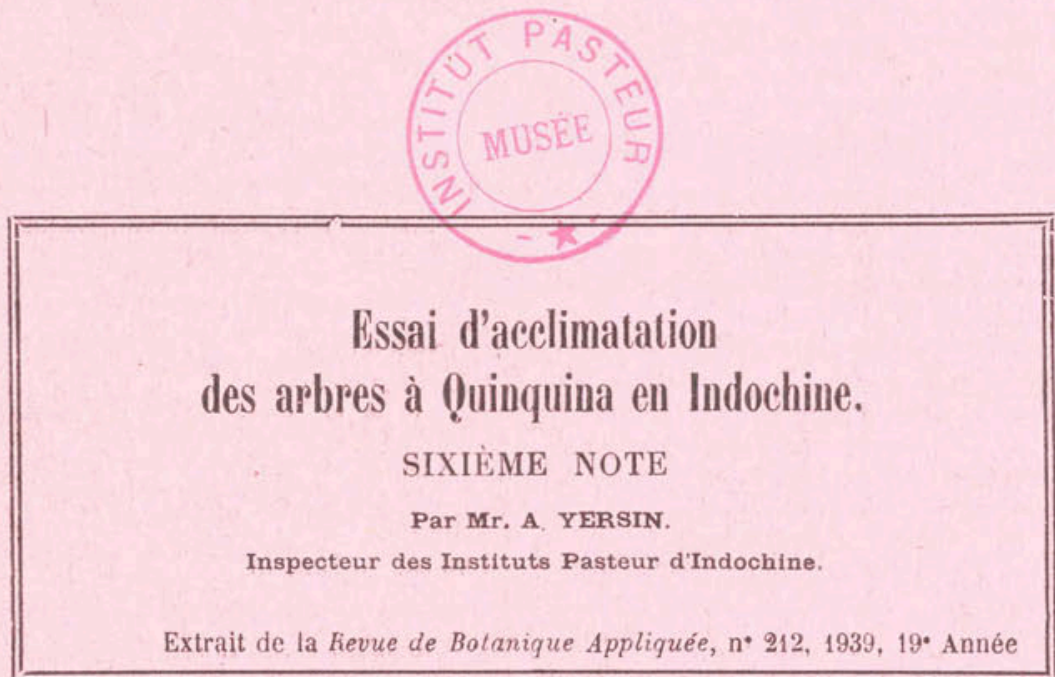

PARIS

Iaboxatoire d'Agromomie coloniale

Au Muséum, 57, rue Cuvier 


\title{
24,744
}

\section{(9) \\ Essais d'acclimatation \\ des arbres à Quninquina en Indochine.}

\author{
SIXIÈME NOTE
}

Par Mr. A. YERSIN.

Inspecteur des Instituts Pasteur d'Indochine.

La dernière note (la cinquième), publiée dans cette Revue par l'Institut Pasteur sur ses essais d'acclimatation des arbres à quinquina en Indochine date de 193 š.

Nous avons eu, depuis, la douleur de perdre en M. Lambert (1) un collaborateur dẻvoué et un ami. Il a largement contribué aux expériences entreprises; les résultats obtenus sont dûs principalement à sa sagacité, à ses connaissances étendues en Chimie, à son ardeur au travail, à son esprit de suite.

La cinquième note traitait principalement des rendements de nos arbres à quinquina suivant leur âge; elle relatait la poursuite d'essais de fumure et donnait quelques indications sur diverses maladies que nous avions constatées dans nos plantations.

Les nouveaux essais ont été poursuivis surtout à la station de Diom

(1) Décédé en France le 3 juillet 4936 à l'àge de 46 ans après une courte maladie. 
(1 000 m. d'altitude), où nous avons entrepris une culture semi-industrielle des arbres à quinquina.

Il ne s'agissait plus d'expériences réduites, sur des petites surfaces, et dont les frais, peu considérables, pouvaient être couverts grâce aux bénéfices réalisés sur la plantation d’Hévéas de Suôi-Giao, appartenant à l'Institut Pasteur de Paris.

Nous avons dû recourir, en vue de financer ces essais nouveaux, à la coupe et à la vente des écorces de la presque totalité des pieds de Quinquinas existant dans nos stations d'essais de Dran, de Djiring, du Petit Lang-Bian et aussi de Diom.

Nous avons recueilli ainsi, en 1936, $29600 \mathrm{~kg}$. d'écorces titrant en moyenne $7,47 \%$ de sulfate de quinine, soit $2045 \mathrm{~kg}$. de sulfate de quinine cristallisé.

La Société du traitement des Quinquinas qui a son siège à Paris, société bien française et qui fait honneur à nos industries nationales a bien voulu s'intéresser, dès le début, à nos essais. Elle nous a assuré une collaboration, un concours technique indispensables. Il convient, en effet, que l'analyse du contenu des écorces, le dosage des alcaloïdes soient effectués dans une usine spécialisée, par comparaison, suivant les mêmes techniques industrielles, avec les écorces de diverses provenances. Les analyses faites sur place, dans la colonie, par les meilleurs techniciens dans les laboratoires les mieux outillés, apportent des indications intéressantes. Elles ne peuvent pas se substituer au contrôle de l'usine.

La Société des Quinquinas a bien voulu prendre en compte nos écorces. Grâce à cet appui nous avons pu entreprendre à Diom des cultures semi-industrielles de $C$. Ledgeriana et les développer sous le seul contrôle qui autorise des conclusions de nature à nous guider sûrement.

Nos prévisions d'exploitation des Quinquinas à Diom (1), sont de $3000 \mathrm{~kg}$. de sulfate de quinine par an (quantité maximum) pour une période de dix ans. Nous ne sommes pas encore organisés pour produire de telles quantités.

En 1937, nous avons obtenu, par les élagages et les éclaircissements à Diom :

Poids d'écorces (net)................... $20.650 \mathrm{~kg}$.

Sulfate de Quinine cristallisé............... 1.434 »

Titre moyen des écorces................. $6,92 \%$

(1) Arrêtées de concert avec la Société du traitement des Quinquinas de Paris. 
En 1938, les résultats ont été, dans les mêmes conditions :

Poids d'écorces....................... $21.100 \mathrm{~kg}$.

Sulfate de Quinine..................... $1.793 \mathrm{~kg} .330$

Titre moyen des écorces................. $8,50 \%$

Pour les années suivantes : $1939,1940 \ldots .$. nous espérons augmenter progressivement la production des écorces, grâce aux extensions en cours, de façon à arriver, dans le plus court délai possible, au rendement annuel de $3000 \mathrm{~kg}$. de sulfate de quinine, quantité maxima prévue pour une période de dix ans.

Voic maintenant les quelques faits nouveaux que nous avons observés à Diom de 1935 à 1938.

Les essais de culture des Quinquinas que nous avons réalisés, en terre naturelle (basaltique) sur le plateau de $1000 \mathrm{~m}$. d'altitude, qui s'étend de Dran à Djiring, nous ont démontré que cette culture est possible, mais que les conditions du sol et du climat, différant notablement de celles qui existent à Java, ne permettent pas aux arbres d'atteindre leur plein développement.

Les plants commencent par se développer normalement, mais la floraison survient déjà la deuxième année, c'est-à-dire beaucoup trop tôt. Dès la fin de la troisième, les pieds, dans une forte proportion, sont atteints d'une maladie des écorces. Elle se manifeste par des lésions du collet : fissures, décollement qui abritent des champignons, Dans la suite, les extrémités des branches se dessèchent et les pieds périssent.

Dans ces conditions, une exploitation industrielle des Quinquinas ne saurait être envisagée que si les arbres étaient coupés dans un cycle limité, à 5 ans par exemple et si la production des écorces de ces arbres peu développés était rémunératrice.

J'ai pensé qu'il serait préférable d'essayer de combattre la maladie et avant tout d'en déterminer les causes. Des observations nombreuses m'ont amené à envisager que l'attaque des pieds au collet par des moisissures (principalement des Fusarium) provenait surtout d'un manque de vitalité et de résistance des Quinquinas. J'ai attribué cette faiblesse aux conditions anormales dans lesquelles nous obligeons les Quinquinas à vivre (climat et sol). Nous ne pouvons pas modifier le climat, mais il est possible d'améliorer les conditions culturales. 
Il paraît évident que des pieds convenablement nourris se défendront mieux que ceux qui seraient contraints à vivre serrés les uns contre les autres dans un sol où ils ne trouveraient pas les aliments qui leur sont nécessaires en quantité suffisante. Il convenait donc de rechercher une formule d'engrais qui apportât au sol les éléments propres à favoriser le développement des Quinquinas.

La culture industrielle des Quinquinas se pratique en plantation serrée : les pieds sont distants de un mètre, ce qui aggrave encore la difficulté pour les plants de s'alimenter suffisamment, dès qu'ils ont atteint un certain développement.

Il convient donc d'élaguer les jeunes plants de toutes les branches basses dès la deuxième année, puis de procéder à des éclaircissements successifs qui permettent d'éliminer, dès la troisième année, tous les pieds malingres, mal venus et de ne laisser se développer que ceux qui paraissent vigoureux. Ceci est d'ailleurs élémentaire.

Nous avons réussi, en procédant ainsi et en répandant abondamment des engrais préparés selon une formule qui comprend un mélange de sels d'azote, de phosphore et de potasse, à donner à nos Quinquinas une vigueur qui doit leur permettre de résister et de vaincre les maladies.

L'enrichissement du sol résultant de l'épandage des engrais a eu aussi pour effet de réveiller de nombreuses souches souterraines d'herbe à paillotte et de Fougères, qui restaient inertes dans la profondeur du sol naturel. Une végétation parasite dense s'est donc développée parallèlement à celle provenant de la croissance des Quinquinas et il a fallu, par des binages soigneux, enlever les souches parasites.

La question qui se pose maintenant est celle-ci : les frais résultant de l'achat des engrais, de leur épandage, des binages nécessaires pour l'élimination des souches parasites seront-ils compensés par l'augmentation du rendement en écorces des Quinquinas? C'est ce que nous ne pouvons pas encore affirmer aujourd'hui.

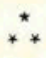

La création de plantations étendues de Quinquinas, sur de grandes surfaces nécessite l'organisation de vastes pépinières. Là aussi nous avons éprouvé des mécomptes :

$1^{\circ}$ Le sol des plates-bandes (terre naturelle), demandait à être enrichi par une fumure appropriée. Nous avons d'abord utilisé du fumier de 
ferme soigneusement pulvérisé et passé au crible. Nous n'avons pas réussi, malgré ces soins, à éliminer les œufs de Coléoptères divers et en particulier de ceux qui sont dénommés « Rhinocéros " et qui dévastent les plantations de Cocotiers. Ces œufs donnent naissance à des chenilles souterraines (vers blancs), qui vivent d'abord dans la profondeur du sol, mais qui ne tardent pas à remonter et à attaquer les plantules des Quinquinas par leurs racines. On voit alors ces plantules s'enfoncer dans le sol, se faner et périr.

Nous avons perdu, du fait des vers blancs, une proportion considérable des plants de semis et nous avons dû renoncer à l'usage du fumier de ferme pour la fumure du sol des plates-bandes. Nous utilisons, dès lors, le même engrais composé que celui qui nous sert pour les surfaces plantées en Quinquinas et nous avons ainsi supprimé cetle cause de non-réussite.

$2^{\circ}$ Pendant la saison des pluies, principalement d'août à fin d'octobre, il pleut énormément à Diom et l'humidité est parfois excessive. Les jeunes plantules ne s'accommodent pas de ces conditions particulières. Elles se trouvent en état de moindre résistance et sont attaquées par les moisissures. Ces moisissures pénètrent dans les tigelles, au niveau du sol, remontent dans leur intérieur, et il en résulte la flétrissure des sommets et la disparition des plantules.

Nous avons perdu ainsi, en 1937 , une proportion considérable des jeunes pieds de semis qui auraient dû être repiqués en 1938. Cette perte a été de $50 \%$ environ. Les conditions défavorables d'humidité excessive et prolongée ne se reproduisent heureusement pas chaque année. Nous espérons y remédier en pratiquant les semis assez tôt pour que les plantules au moment des grosses pluies, soient mieux développées et plus résistantes. Nous les espacerons davantage. Nous envisageons également de prévoir, en faisant les semis, une marge de sécurité pour une perte possible de $50 \%$ sur les pieds à repiquer et augmenter en proportion la surface des plates-bandes de semis.

Nous pouvons conclure de cet exposé que la période expérimentale de nos essais d'acclimatation des Quinquinas en Indochine est loin d'être close. Nous constatons chaque jour des faits nouveaux; nous nous trouvons en face de difficultés imprévues ; mais nous avons l'espoir de remédier aux aléas qui se produiront encore par des dispositions appropriées que nous suggérera l'expérience. 
- Il convient d'être en garde contre le désir de réalisations immédiates fondées sur des résultats fragmentaires qui seraient le point de départ de déceptions ultérieures et nuiraient à l'œuvre entreprise au lieu de la servir. Vingt années d'études désintéressées poursuivies avec continuité et dans le même souci d'objectivité, nous ont convaincu du danger des conclusions hâtives aussi bien dans le sens d'un découragement prématuré que de trompeuses illusions. Partout où il se posera, le problème de l'acclimatation de l'arbre à Quinquina ne sera résolu qu'à la suite d'un effort mélhodique et persévérant soumis à un contrôle judicieux et permanent. 